\title{
O.S.P.
}

L'orientation scolaire et professionnelle

$39 / 4 \mid 2010$

varia

\section{L'évaluation de l'indécision vocationnelle : l'EDV-9} (forme S)

Assessing vocational indecision : the EDV-9 (S form)

Yann Forner

(2) OpenEdition

Journals

Édition électronique

URL : http://journals.openedition.org/osp/2968

DOI : $10.4000 /$ osp.2968

ISSN : 2104-3795

Éditeur

Institut national d'étude du travail et d'orientation professionnelle (INETOP)

Édition imprimée

Date de publication : 31 décembre 2010

ISSN : 0249-6739

Référence électronique

Yann Forner, «L'évaluation de l'indécision vocationnelle : I'EDV-9 (forme S) », L'orientation scolaire et professionnelle [En ligne], 39/4 | 2010, mis en ligne le 31 décembre 2013, consulté le 22 octobre 2020 URL : http://journals.openedition.org/osp/2968 ; DOI : https://doi.org/10.4000/osp.2968

Ce document a été généré automatiquement le 22 octobre 2020

(c) Tous droits réservés 


\title{
L'évaluation de l'indécision vocationnelle : l'EDV-9 (forme S)
}

Assessing vocational indecision : the EDV-9 (S form)

\author{
Yann Forner
}

1 L'indécision est l'incapacité d'une personne à exprimer un choix pour une activité différenciée lorsqu'elle est incitée à le faire. On fait état d'indécision vocationnelle lorsque le choix porte sur la future activité professionnelle ou sa préparation.

2 L'épreuve permet, d'une part, d'estimer l'intensité de l'état d'indécision vocationnelle telle qu'il est ressenti par la personne et l'on distinguera l'indécision professionnelle (celle qui porte sur la fin, le métier) et l'indécision scolaire (celle qui porte sur le moyen, la formation). L'épreuve permet, d'autre part, d'évaluer l'importance de facteurs susceptibles d'expliquer cet état d'indécision.

3 L'épreuve existe sous deux formes chacune adaptée à un type principal d'utilisation. La forme $\mathrm{S}$ (scolaire) est plus pertinente pour les activités collectives d'aide à l'orientation menées, pour l'essentiel, dans le cadre de l'enseignement secondaire: il s'agit alors, soit d'estimer les besoins de groupes pour mieux ajuster les interventions des équipes éducatives, soit d'évaluer à quel point ces besoins ont été satisfaits. La forme I (insertion) est plus adaptée aux consultations d'orientation et des bilans individuels: il s'agit de déterminer la nature de l'aide à apporter aux personnes indécises pour la construction de projets personnels de carrière. Dans sa forme $S$, celle qui est présentée ici, l'épreuve est particulièrement destinée aux jeunes de l'enseignement secondaire: de la classe de quatrième de collège, voire de cinquième pour les collégiens ayant un niveau verbal suffisant, jusqu'au premier cycle de l'enseignement supérieur. Elle permet d'estimer les poids relatifs de six facteurs dans la détermination de l'indécision: manque de développement, manque de connaissance de soi, manque d'information sur le monde vocationnel, absence de méthode de décision, anticipations pessimistes et obstacles externes.

4 L'épreuve comporte 55 items (forme S) dont 5 permettant d'évaluer l'état d'indécision tel qu'il est perçu, les autres items permettant d'estimer les poids respectifs des six 
déterminants considérés. Elle peut être passée en situation individuelle ou collective. Le temps de passation est libre; en règle générale, il est de l'ordre de 15 à 20 minutes, temps de lecture des consignes compris.

5 L'épreuve et son manuel (Forner, 2009) sont disponibles aux Éditions Eurotests (www.eurotetsts.fr); le présent article résume la partie consacrée à la forme $\mathrm{S}$ de l'épreuve.

\section{Fondements théoriques}

6 On peut distinguer quatre grandes conceptions de l'indécision vocationnelle parmi celles qui ont guidé l'élaboration de l'EDV-9: l'absence de choix, l'état transitoire d'élaboration d'un choix, le dysfonctionnement des processus de choix et la multidétermination potentielle.

\section{L'absence de choix}

7 Pour certains (pour une analyse plus précise et des références, on se reportera aux écrits signalés en bibliographie), on peut faire état d'indécision de carrière quand une personne éprouve des difficultés à arrêter un choix de carrière. Cette conception élargie de l'indécision semble fréquemment adoptée depuis plus d'une décennie. Ce n'était pas celle des premières études où l'indécision était simplement définie par l'absence de réponse d'une personne à un questionnement sur ses intentions d'avenir en matière professionnelle. Cette approche syncrétique, que l'on pourrait qualifier d'administrative, incite à considérer également indécises des personnes qui n'ont en commun que leur état d'indécision, sans qu'une hypothèse ne soit formulée quant aux causes de cet état. Au terme d'études portant souvent sur de grands effectifs, on parvenait à une synthèse décevante plutôt: très généralement jeunes décidés et jeunes indécis ne se différencient quasiment pas. La déception était à la mesure des attentes, car le problème devenait important avec l'allongement et la spécialisation des formations, et à la mesure des moyens investis dans les recherches. Des études plus récentes peuvent également être rattachées à cette approche dichotomique: celles où l'on substitue un continuum de décision - indécision à la simple opposition décidé/ indécis. On y établit des corrélations entre la dimension indécision et d'autres dimensions, comme la planification, l'exploration professionnelle, le développement de l'identité ou l'estime de soi (corrélations négatives), la timidité, la peur de réussir ou l'externalité du contrôle (corrélations positives). Cette démarche a été particulièrement utilisée pour des dimensions de personnalité comme l'anxiété, la peur de l'engagement, l'introversion ou la dépression.

\section{Le moratoire: l'état transitoire d'élaboration de la décision à l'adolescence}

Le relatif échec de ces premières approches était compatible avec les conceptions développementales selon lesquelles, une fois passées les rêveries de l'enfance, les décisions d'orientation ne peuvent se prendre qu'à l'issue d'un long processus. Il en résulterait l'existence, en cours d'adolescence, d'une nécessaire période d'élaboration du choix et, donc, d'indécision. Comme les personnes quittent cette période à des âges 
(chronologiques) différents, ces décalages font apparaître décidées les personnes dont le développement est précoce, et indécises celles dont le développement est tardif. Dans cette conception développementale, on a parfois fait de la tendance à décider (par opposition à l'indécision) une dimension de la maturité vocationnelle, un état préparatoire aux prises de décision de carrière. Ce nouveau modèle a été conforté par diverses études, mais il laissait apparaître une difficulté: tous les états indécis ne décroissent pas lors de l'adolescence. L'indécision n'est plus alors un inévitable état transitoire de l'adolescence, mais une caractéristique de la personne. Cette première différenciation permettait d'expliquer, en partie, la faiblesse voire l'inconsistance des contrastes entre les personnalités des jeunes décidés et des jeunes indécis, ces derniers ne formant pas un groupe homogène. On repérait donc une forme ancrée d'indécision: l'indécision chronique. Caractéristique des personnes, elle était censée se manifester dans d'autres situations que les décisions de carrière, c'est pourquoi on a fait également état d'indécision généralisée. Pour certains, ce serait la manifestation d'une personnalité indécise, l'association d'un manque de maturité vocationnelle (manque de connaissance de soi et des milieux professionnels) et d'insuffisances moins développementales (comme le manque de confiance en soi ou l'intolérance à l'ambiguïté). D'autres ont envisagé l'existence de deux voies de développement vocationnel à l'adolescence: l'une normale, et l'autre (marquée par une forte anxiété) se manifestant par des perturbations du processus de décision notamment la tendance à la forclusion et la peur de l'engagement).

\section{Le dysfonctionnement des processus de choix}

Des conceptions cognitives de l'indécision ont complété l'approche développementale: elles visaient à déterminer formes et contenus des phases du traitement nécessaire de l'information concernant la personne elle-même, les métiers, le monde du travail et celui des formations. Un modèle de décision prévoyait cinq phases, chacune caractérisée par l'utilisation dominante d'un processus: communication (prélèvement d'information), analyse (exploration), synthèse (cristallisation), évaluation et exécution. Ces traitements de l'information sont contrôlés par des règles métacognitives plus ou moins pertinentes (une règle non pertinente serait, par exemple, celle selon laquelle il n'y aurait qu'une seule profession satisfaisante pour soi). Dans ces approches, qui sont particulièrement représentées dans le monde francophone par la démarche A.D.V.P., l'indécision n'est pas considérée spécifiquement mais traitéecomme la conséquence du dysfonctionnement de l'un, au moins, des processus de décision. Cette conception normative est opérationnelle mais elle mène les auteurs à étendre la notion d'indécision. Certains, en effet, considèrent décidées les seules personnes qui ont exercé de manière approfondie les processus requis: en conséquence, un jeune qui indiquerait un choix unique et réaliste, mais formulé à l'issue d'un traitement superficiel de l'information, serait un «faux positif», artificiellement décidé! Une telle fidélité à la logique des processus risque d'éloigner de la réalité des comportements: un jeune peut décider à l'aide d'un processus insuffisant; on n'a pas montré qu'il s'adaptera différemment d'un autre au monde professionnel.

10 La diversité potentielle des processus. L'opposition initiale entre indécision développementale et indécision généralisée a préfiguré diverses distinctions. Ainsi, l'insuffisance de l'information sur soi ou sur les professions a pu être associée à d'autres états d'indécision. Il s'agit souvent d'une insuffisance subjectivement ressentie, car il 
n'apparaît pas que la tendance à choisir et la sûreté du choix augmentent mécaniquement avec la quantité d'information dont on dispose. L'existence d'un état indécis a également été associée à celle d'un conflit interne (il s'agit ici d'un conflit du type approche, celui éveillé par la coexistence de plusieurs buts également attirants, mais mutuellement exclusifs). Pour une formalisation plus opérationnelle de cette forme d'indécision, on a caractérisé les personnes par leur absence de méthode de décision, suggérant que la mise à disposition d'une méthode les amène à se déterminer. On est ainsi passé d'une analyse de deux formes possibles d'indécision à l'analyse de l'importance, chez un même sujet, de ses principaux facteurs potentiels et on a fait état d'un diagnostic différentiel de l'indécision. Cette approche, plus fine que les précédentes, nécessitait des outils de mesure plus systématiques et précis et plusieurs échelles ont été élaborées. Les réponses à ces questionnaires ont été traitées à l'aide d'analyses factorielles dont les dimensions sont devenues des déterminants potentiels de l'indécision.

\section{L'évolution de l'épreuve}

\section{Le questionnaire Décision - Indécision}

Dans ce questionnaire on distingue l'intensité perçue de l'état d'indécision et les facteurs individuels de son installation. L'importance subjective de l'état d'indécision est appréciée à l'aide de deux questions dont l'une porte sur l'élaboration d'un choix d'étude ou de formation, et l'autre sur un choix de métier ou d'activité professionnelle. Pour chacune, le jeune indique l'intensité de son indécision en se repérant en fonction des quatre situations proposées. Les scores, croissants de 0 à 3 pour chaque question, peuvent être additionnés pour fournir un indice total d'indécision (variant donc de 0 à 6). Les facteurs d'indécision considérés sont au nombre de huit, appréciés chacun à l'aide de cinq items. 1) Obstacles externes (exemple d'items: «J'ai découvert que je ne pourrai pas exercer le métier qui m’intéresse»), 2)Indécision développementale («Je me préoccupe encore assez peu de mon avenir professionnel»), 3) Indécision généralisée («Je retarde toujours autant que je peux la prise de toute décision»), 4) Absence de méthode («Plusieurs métiers me semblent également attirants»), 5) Anxiété dans la décision («Devoir choisir un métier me met très mal à l'aise»), 6) Manque d'information («Je manque d'information sur les professions»), 7) Anticipations pessimistes ( J'ai peur de ne pas réussir dans les études que je voudrais faire»), 8) Désinvestissement du travail («Je trouve que faire des études ne me servirait à rien»). Formulation initiale, ce questionnaire n'a plus lieu d'être utilisé.

\section{Étude et nouvelle formulation de l'instrument}

Une recherche (Forner, 1995) visait à éprouver les qualités de cette première forme du questionnaire et du modèle sous-jacent, puis à dégager des indications pour d'éventuels aménagements. Les principaux résultats sont les suivants: 1) l'intensité de l'indécision a été précisée. 2) Les fréquences des causes d'indécision sont très diverses: le manque d'information est très courant; le désinvestissement du travail est rare. 3)La structure des relations entre les items incite à reconsidérer certains facteurs: notamment, les dimensions Indécision généralisée et Anxiété de décision apparaissent quasiment indissociables. À l'inverse, la formulation du Désinvestissement du travail manque de 
cohérence (on distingue des items dont le contenu est scolaire et d'autres dont le contenu est professionnel).

\section{La précédente Échelle de Décision Vocationnelle, EDV}

13 Comme précédemment, le sujet doit estimer son niveau d'indécision, puis répond à 32 items à partir desquels on pourra déterminer ses scores sur les facteurs suivants: 1)Personnalité («J'ai peur que mon choix professionnel ne soit pas le bon»), 2)Développement ( Je n'ai pas besoin de choisir dès maintenant ce que je ferai plus tard»), 3) Méthode («Je manque de méthode pour choisir les études qui me plairaient»), 4)Information («Je manque d'informations sur le monde du travail»), 5) Obstacles externes («Mes projets sont bloqués à cause de mes résultats scolaires»). 6)Anticipations pessimistes («Je crains de ne pas parvenir au métier qui m’intéresse»), 7)Désinvestissement scolaire ( «Je trouve que toutes les formations sont sans intérêt pour moi»).

\section{L'actuelle Épreuve de Décision Vocationnelle-9 forme scolaire: l'EDV-9s}

14 L'organisation de l'épreuve est inchangée: après s'être identifié, l'utilisateur doit d'abord estimer son indécision scolaire, puis, il doit expliciter sa réponse. Il doit alors estimer son indécision professionnelle et doit, de même, expliciter sa réponse. Puis il doit répondre à 48 items, chacun de ces items correspondant à un facteur censé permettre d'expliquer l'état indécis. Les facteurs considérés ici sont: 1) un développement tardif en matière vocationnelle, 2)le manque d'information sur le monde vocationnel, 3)le manque de connaissance de soi, 4)le manque de méthode pour décider, 5)des obstacles externes qui empêchent la réalisation d'un projet et 6)des anticipations trop pessimistes pour permettre la constitution d'un projet.

Cette forme $\mathrm{S}$ se double d'une forme I non présentée ici.

\section{L'utilisation de l'épreuve}

\section{Contexte d'utilisation}

16 L'épreuve concerne les adolescents et jeunes adultes qui éprouvent des difficultés dans l'élaboration de leurs projets de formation et d'emploi. Elle s'adresse, d'une part, aux personnes qui fréquentent l'enseignement secondaire ou le début de l'enseignement supérieur et vise plus particulièrement les jeunes des lycées, qu'ils suivent un enseignement général ou technologique, voire professionnel. Elle peut être utilisée à partir de la classe de quatrième de collège jusqu'au premier cycle universitaire. L'épreuve s'adresse, d'autre part, aux personnes engagées dans une activité de bilan: bilan de compétences personnelles et professionnelles, bilan de réinsertion professionnelle des demandeurs d'emploi, bilan mené lors de stages d'insertion ou de reconversion. Pour ces dernières situations, la forme EDV-9 I est sans doute plus adaptée. 


\section{Consignes pour la passation}

17 Elles figurent sur la première page de la feuille de passation. Il convient de les lire à voix haute aussi strictement que possible. La lecture devra être d'autant plus rigoureuse que le niveau de formation des personnes sera faible. On pourra adapter cette lecture pour les personnes de niveau de formation élevé (pour plus d'indications, on se reportera au manuel de l'épreuve).

\section{Consignes pour le dépouillement}

18 Pour les évaluations de l'état d'indécision, les scores bruts du sujet sont les chiffres correspondant à ses réponses. Le score brut d'indécision vocationnelle est la somme des deux scores précédents. L'étalonnage se limite à des indications sur le rang du sujet (voir tableau 7). Pour les évaluations des facteurs de l'indécision, il faut établir les scores bruts sur chaque échelle en sommant les notes obtenues aux 8 items des échelles avec $A=1 ; B=2 ; C=3$ et $D=4$. L'étalonnage est un décilage (voir tableau 8). On trouvera dans le manuel des étalonnages différenciés selon le genre.

\section{Indications pour la restitution}

19 L'Épreuve de Décision Vocationnelle est d'abord un outil destiné au spécialiste du conseil: cet outil lui indique un mode de réponse spécifique à une personne indécise et lui fournit un guide pour un entretien plus approfondi. Mais ce peut être également un outil psychopédagogique destiné à la personne indécise pour lui permettre de prendre conscience de la représentation qu'elle a de sa propre situation, d'élaborer cette représentation et de la faire évoluer. En ce sens il faut fournir une information synthétique de ses déclarations au jeune (pour plus d'indications, on se reportera au manuel de l'épreuve).

\section{Caractéristiques techniques de l'épreuve}

\section{Population et échantillon}

20 La population est celle des jeunes de l'enseignement secondaire. Une enquête a été menée auprès d'élèves de classes de première $(N=642)$, ainsi qu'auprès d'élèves de classes de troisième $(N=510)$. L'échantillon retenu comporte donc 1152 participants: 455 garçons $(39,5 \%)$ et 697 filles (60,5\%). Les âges variaient de 12 à 20 ans autour d'une moyenne de 15,62 ans avec un écart-type de 1,35 an. Les participants ont été interrogés collectivement, dans le cadre scolaire traditionnel, au cours des deuxième et troisième trimestres scolaires.

\section{Analyse des items}

21 La distribution des scores d'indécision scolaire (question A) figure au tableau 1. En matière de formation, un jeune sur sept $(13,5 \%)$ est totalement indécis et un sur trois $(35,6 \%)$ hésite entre plusieurs voies. Les pourcentages ont été cumulés à partir des plus faibles scores, c'est-à-dire des sujets les plus décidés. La distribution des scores 
d'indécision professionnelle (question C) figure au tableau 2. En matière d'activité professionnelle, un jeune sur six $(16,7 \%)$ est totalement indécis et un sur trois $(30,8 \%)$ hésite entre plusieurs activités. La distribution des scores d'indécision vocationnelle figure au tableau 3. En matière de carrière, un jeune sur cinq $(12,2 \%+3,9 \%=16,1 \%)$ est totalement décidé au moins sur une des deux échelles et un sur six $(20,4 \%)$ est totalement indécis au moins sur une des deux échelles.

Quarante-huit items décrivent des causes potentielles de l'état (évalué par les précédents items). La description de ces items figure en annexe I.On note l'asymétrie de la distribution de 5 items, relevant tous de l'échelle Obstacles. L'étude de cette asymétrie sera reprise, ci-dessous, dans l'étude des échelles.

Tableau 1

L'indécision scolaire dans l'enseignement secondaire $(\mathrm{N}=1152)$

\begin{tabular}{|l|c|c|c|c|}
\hline $\begin{array}{l}\text { Ensemble } \\
(\mathrm{n}=1152)\end{array}$ & $\begin{array}{c}\text { Une voie } \\
\text { définitivement fixée }\end{array}$ & $\begin{array}{c}1 \\
\text { Une voie } \\
\text { non définitive }\end{array}$ & $\begin{array}{c}2 \\
\text { Plusieurs voies } \\
\text { envisagées }\end{array}$ & $\begin{array}{c}3 \\
\text { Aucune idée } \\
\text { précise }\end{array}$ \\
\hline Pourcentages & 19.3 & 31.5 & 35.6 & 13.5 \\
\hline \% cumulés & 19.3 & 50.8 & 56.4 & $(100)$ \\
\hline
\end{tabular}

\section{Tableau 2}

L'indécision professionnelle dans l'enseignement secondaire ( $\mathrm{N}=1152)$

\begin{tabular}{|l|c|c|c|c|}
\hline $\begin{array}{l}\text { Ensemble } \\
(\mathrm{n}=1152)\end{array}$ & $\begin{array}{r}\text { Un métier fixé } \\
\text { définitivement }\end{array}$ & $\begin{array}{c}1 \\
\text { Un métier } \\
\text { non fixé }\end{array}$ & $\begin{array}{c}2 \\
\text { Plusieurs } \\
\text { métiers }\end{array}$ & $\begin{array}{c}3 \\
\text { Aucune idée } \\
\text { précise }\end{array}$ \\
\hline Pourcentages & 21.6 & 30.8 & 30.9 & 16.7 \\
\hline \% cumulés & 21.6 & 52.4 & 83.3 & $(100)$ \\
\hline
\end{tabular}

Tableau 3

L'indécision vocationnelle dans l'enseignement secondaire

\begin{tabular}{|l|c|c|c|c|c|c|c|}
\hline & 0 & 1 & 2 & 3 & 4 & 5 & 6 \\
\hline Pourcentages & 6.6 & 13.8 & 20.3 & 25.7 & 17.6 & 12.2 & 3.9 \\
\hline \% cumulés & 6.6 & 20.4 & 40.7 & 66.4 & 84.0 & 96.2 & $(100,0)$ \\
\hline
\end{tabular}




\section{La structure des relations entre items} rotation Varimax figure au tableau 4. L'hypothèse est celle d'un regroupement des items sur 6 composantes correspondant aux échelles précédentes. Ces 6 composantes expliquent près de la moitié $(48,05 \%)$ de la variance du questionnaire. (N.B. On a utilisé ici systématiquement le terme "composante» pour désigner chacune des dimensions issues de l'analyse factorielle et le terme «facteur» pour désigner chacune des causes potentielles d'indécision évaluées par le questionnaire). La première composante ( $\mathrm{C} 1$ dans le tableau 4) sature les items $30,41,13,7,46,36,02$, 19; elle explique $20,86 \%$ de la variance et peut être interprétée comme une composante de manque de développement. La seconde composante (C2) sature les items 29, 24, 12, 6, 1, 32, 45, 18, 15 elle explique $8,49 \%$ de la variance et peut être interprétée comme une composante de manque de Méthode. La troisième composante sature les items 15, 03, 09, 26, 24, 38, 16, 39; elle explique $6,66 \%$ de la variance et peut être interprétée comme une composante d'anticipations pessimistes. La quatrième composante sature les items 44 , $40,17,11,33,28,23$, 47; elle explique $4,84 \%$ de la variance et peut être interprétée comme une composante de manque d'Information. La cinquième composante sature les items $10,27,05,22,48,43,35,21$; elle explique $3,66 \%$ de la variance et peut être interprétée comme une composante de manque de connaissance de Soi. La sixième composante sature les items $20,08,03,37,31,42,14,25$; elle explique $3,54 \%$ de la variance et peut être interprétée comme une composante d'obstacles externes.

Ces composantes sont orthogonales, elles correspondent à l'idée de 6 sources de variation indépendantes (i.e. un score observé pour un sujet sur une composante ne permet aucun pronostic sur le score de ce sujet sur les autres composantes). L'organisation des saturations présentées au tableau 4 permet de conforter l'hypothèse selon laquelle l'état d'indécision des jeunes de l'enseignement secondaire relève de six principales sources indépendantes.

Tableau 4

Analyse factorielle exploratoire sur les items

Rotation Varimax - N = 1152 élèves de l'enseignement secondaire

Les saturations inférieures à .20 ont été supprimées

\begin{tabular}{|l|l|l|l|l|l|l|}
\hline & $\mathrm{C} 1$ & $\mathrm{C} 2$ & $\mathrm{C} 3$ & $\mathrm{C} 4$ & $\mathrm{C} 5$ & $\mathrm{C} 6$ \\
\hline $\mathrm{V} 30$ &, 798 & & & & & \\
\hline $\mathrm{V} 41$ &, 780 & & & & & \\
\hline $\mathrm{V} 13$ &, 769 & & & & & \\
\hline $\mathrm{V} 07$ &, 758 & & & & & \\
\hline $\mathrm{V} 46$ &, 725 & & & & & \\
\hline
\end{tabular}




\begin{tabular}{|c|c|c|c|c|c|c|}
\hline V36 & ,703 & & & & & \\
\hline V02 & 697 & & & & & \\
\hline V19 & 696 & & & & & \\
\hline V29 & & 855 & & &,- 243 & \\
\hline V24 & &, 780 & & & & \\
\hline V12 & &, 758 & & & & \\
\hline V06 & & ,756 & & & & \\
\hline V01 & &, 750 & & & & \\
\hline V32 & & ,603 & & & & \\
\hline V45 & &, 578 & & & & \\
\hline V18 & & ,463 & & ,227 & 296 & \\
\hline V15 & & & ,838 & & & \\
\hline V04 & & & ,794 & & & \\
\hline V09 & & & ,716 & & & \\
\hline V26 & & & ,713 & & & \\
\hline V34 & & & 690 & & & \\
\hline V38 & & &, 533 & & & \\
\hline V16 & & &, 525 & & & 259 \\
\hline V39 & & & ,449 & & 271 & \\
\hline V44 & & & & ,744 & & \\
\hline V40 & & & & ,718 & & \\
\hline V17 & & & &, 712 & & \\
\hline V11 & & & & ,700 & & \\
\hline V33 & & & & 639 & & \\
\hline $\mathrm{V} 28$ & & & & ,610 & & \\
\hline V23 & & & & 570 & & \\
\hline
\end{tabular}




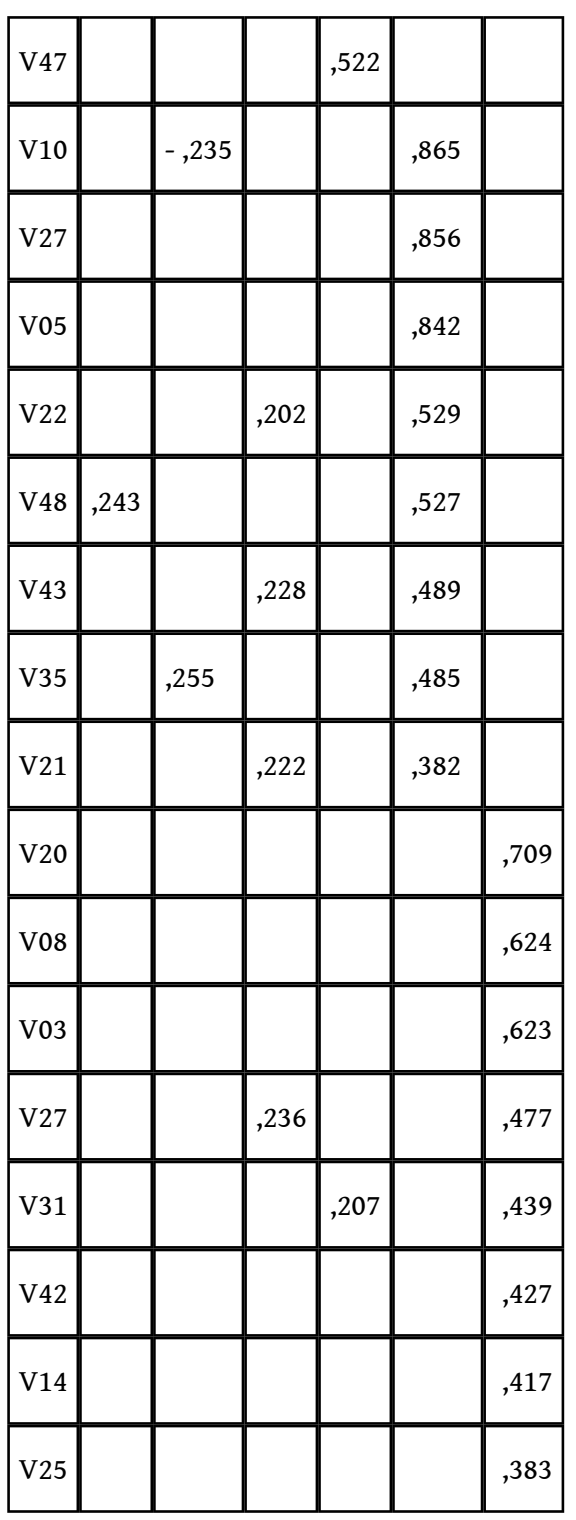

\section{Analyse des échelles}

31 L'unidimensionnalité des échelles a été examinée en appliquant une analyse factorielle (suivie de la rotation Varimax) aux items de chaque échelle. L'hypothèse d'unidimensionnalité ne paraît jamais prise en défaut. Pour chaque échelle on trouvera (tableau 5) divers indices caractérisant la distribution des scores: tendance centrale (la moyenne), dispersion (l'écart-type), normalité (le coefficient de Kolmogorov-Smirnov), forme (les coefficients d'asymétrie et d'aplatissement) et homogénéité (coefficient alpha de (ronbach). Pour préciser l'homogénéité on trouvera au tableau 6, pour chaque item, le coefficient de corrélation avec son échelle de rattachement (coefficient corrigé pour l'appartenance de l'item à l'échelle).

N.B.:

-L'asymétrie des distributions est évaluée par un coefficient de skewness fondé sur le rapport du moment centré d'ordre 3 au cube de l'écart-type. Ce coefficient est négatif lorsque la distribution est plus dispersée pour les faibles valeurs de la variable; il est nul lorsque la 
distribution est symétrique; il est positif lorsque la distribution est plus dispersée pour les fortes valeurs de la variable.

- L'aplatissement des distributions est évalué par un coefficient de kurtosis fondé sur le rapport du moment centré d'ordre 4 au carré de la variance. Ce coefficient est négatif lorsque les valeurs extrêmes sont plus rares que dans une distribution gaussienne; il est nul pour une distribution strictement gaussienne; il est positif lorsque les valeurs extrêmes sont plus fréquentes que dans une distribution gaussienne.

- L'homogénéité des échelles est appréciée par le coefficient alpha de Cronbach, qui est fonction du nombre d'items de l'épreuve: ici, malgré la brièveté des échelles (8 items), on note que les indices d'homogénéité sont satisfaisants.

- La normalité des distributions des échelles est évaluée par test de Kolmogorov-Smirnov. On note ici qu'aucune distribution n'est strictement normale, mais pas au point de ne pouvoir utiliser les statistiques paramétriques (sur ce point on peut voir http:// www.physics.csbsju.edu/stats/KS-test.html).

Tableau 5

\begin{tabular}{|l|c|c|c|c|c|c|}
\hline Ėchelles & Développement & \multicolumn{1}{|c|}{ Soi } & Information & Méthode & Anticipations & Obstacles \\
\hline Minimum & 13.48 & 1118 & 118 & 1118 & 118 & 1118 \\
\hline Maximum & 13.32 & 11132 & 32 & 32 & 32 & 29 \\
\hline Moyenne & 13.49 & 17.51 & 19.62 & 18.56 & 18.97 & 10.59 \\
\hline Ėcart-type & 5.27 & 5.96 & 5.63 & 6.10 & 5.43 & 3.03 \\
\hline Aplatissement & .69 & -.71 & -.75 & -.88 & -.63 & 4.05 \\
\hline Asymétrie & 1.07 & .36 & -.01 & .11 & .08 & 1.75 \\
\hline Normalité & 5.09 & 2.96 & 2.03 & 1.95 & 1.84 & 6.70 \\
$(\mathrm{p}<.01)$ & $(\mathrm{p}<.01)$ & $(\mathrm{p}<.01)$ & $(\mathrm{p}<.01)$ & $(\mathrm{p}<.01)$ & $(\mathrm{p}<.01)$ \\
\hline Homogénéité & .867 & .855 & .825 & .848 & .835 & .657 \\
\hline
\end{tabular}

Tableau 6

\begin{tabular}{|l|l|l|l|l|l|l|l|l|}
\hline Items & V02 & V07 & V13 & V19 & V30 & V38 & V41 & V46 \\
\hline $\mathrm{r}$ & .568 & .593 & .656 & .630 & .687 & .566 & .658 & .595 \\
\hline
\end{tabular}




\begin{tabular}{|l|l|l|l|l|l|l|l|l|}
\hline Items & V05 & V10 & V21 & V22 & V27 & V35 & V43 & V48 \\
\hline $\mathrm{r}$ & .592 & .562 & .505 & .604 & .689 & .611 & .568 & .646 \\
\hline
\end{tabular}

37 Échelle « Information »

\begin{tabular}{|l|l|l|l|l|l|l|l|l|}
\hline Items & V11 & V17 & V23 & V28 & V33 & V40 & V44 & V47 \\
\hline $\mathrm{r}$ & .505 & .586 & .556 & .522 & .549 & .586 & .633 & .551 \\
\hline
\end{tabular}

Échelle « Méthode »

\begin{tabular}{|l|l|l|l|l|l||l|l|l|}
\hline Items & V01 & V06 & V12 & V18 & V24 & V29 & V32 & V45 \\
\hline $\mathrm{r}$ & .553 & .635 & .640 & .585 & .585 & .648 & .625 & .422 \\
\hline
\end{tabular}

Échelle « Anticipations »

\begin{tabular}{|l|l|l|l|l|l|l|l|l|}
\hline Items & V04 & V09 & V15 & V16 & V26 & V34 & V38 & V39 \\
\hline $\mathrm{r}$ & .614 & .595 & .691 & .461 & .644 & .562 & .439 & .497 \\
\hline
\end{tabular}

Échelle « Obstacles »

\begin{tabular}{|l|c|c|c|c|c|c|c|c|}
\hline Items & V03 & V08 & V14 & V20 & V25 & V31 & V37 & V42 \\
\hline $\mathrm{r}$ & .365 & .319 & .252 & .416 & .328 & .311 & .402 & .393 \\
\hline
\end{tabular}

\section{Sources de variabilité}

\section{Niveau de formation}

41 Une comparaison simple des scores moyens des collégiens de troisième et des lycéens de première montre:

- que pour quatre comparaisons, les scores des collégiens sont les plus élevés: indécision vocationnelle, manque de développement, manque de connaissance de soi et obstacles externes;

- que pour les trois autres comparaisons, ce sont les scores des lycéens qui dominent: manque de méthode, manque d'information et anticipations pessimistes; 
- qu'aucune de ces différences n'est significative.

On peut conclure à l'absence d'effet simple du niveau de formation sur l'indécision et ses facteurs.

\section{Genre des participants}

Garçons et filles présentent en moyenne des états d'indécision comparables $(F=.98$; n.s.), mais ils ne sont pas indécis pour les mêmes raisons: l'indécision des garçons est plutôt le fait d'un manque de développement $(F=58,76 ; p<.001)$, l'indécision des filles étant plus marquée par des anticipations pessimistes $(F=40,24 ; p<.001)$, un manque de connaissance de soi $(F=21.63 ; p<.001)$ et, peut-être, un manque de méthode $(F=5.74 ; p$ $<.02$ ). Bien que significatives, ces différences restent d'ampleur très modeste et n'imposent pas l'usage d'étalonnages différenciés.

\section{Âge des participants}

Les corrélations entre l'âge et les variables d'indécision sont toujours très faibles (comprises entre -.10 et +.10 ) et jamais significatives: -.06 (pour l'indécision vocationnelle), -.05 (manque de développement), -.02 (manque de méthode), +.05 (anticipations pessimistes), +.01 (manque d'information), -.05 (manque de connaissance de soi), - .01 (obstacles externes). On peut conclure à l'absence d'effet simple de l'âge sur l'indécision et ses facteurs.

\section{Étalonnages}

Tableau 7

L'indécision dans l'enseignement secondaire $(\mathrm{N}=1152)$

Indécision scolaire

\begin{tabular}{|l|c|c|c|c|}
\hline Ensemble & 0 & 1 & 2 & 3 \\
$(\mathrm{n}=1152)$ & Une voie & $\begin{array}{c}\text { Une voie } \\
\text { définitivement fixée }\end{array}$ & $\begin{array}{c}\text { Non définitive } \\
\text { envisagées }\end{array}$ & $\begin{array}{c}\text { Pucune idée } \\
\text { précise }\end{array}$ \\
\hline Pourcentages & 19.3 & 31.5 & 35.6 & 13.5 \\
\hline Rangs & $1^{\mathrm{er}}$ & $20^{\mathrm{e}}$ & $51^{\mathrm{e}}$ & $87^{\mathrm{e}}$ \\
\hline
\end{tabular}

Indécision professionnelle 


\begin{tabular}{|l|c|c|c|c|}
\hline Ensemble & 0 & 1 & 2 & 3 \\
$(\mathrm{n}=1152)$ & Un métier fixé & Un métier & Plusieurs & Aucune idée \\
pritivement & non fixé & métiers & préce \\
\hline Pourcentages & 21.6 & 30.8 & 30.9 & 16.7 \\
\hline Rangs & $1^{\mathrm{er}}$ & $22^{\mathrm{e}}$ & $53^{\mathrm{e}}$ & $84^{\mathrm{e}}$ \\
& $21^{\mathrm{e}}$ & $52^{\mathrm{e}}$ & $83^{\mathrm{e}}$ & $100^{\mathrm{e}}$ \\
\hline
\end{tabular}

Indécision vocationnelle

\begin{tabular}{|l|c|c|c|c|c|c|c|}
\hline $\begin{array}{l}\text { Ensemble } \\
(\mathrm{n}=1152)\end{array}$ & 0 & 1 & 2 & 3 & 4 & 5 & 6 \\
\hline Pourcentages & 6.6 & 13.8 & 20.3 & 25.7 & 17.6 & 12.2 & 3.9 \\
\hline Rangs & \multicolumn{1}{|c|}{$1^{\mathrm{er}}$} & $7^{\mathrm{e}}$ & $21^{\mathrm{e}}$ & $41^{\mathrm{e}}$ & $67^{\mathrm{e}}$ & $85^{\mathrm{e}}$ & $97^{\mathrm{e}}$ \\
\hline & $6^{\mathrm{e}}$ & $20^{\mathrm{e}}$ & $40^{\mathrm{e}}$ & $66^{\mathrm{e}}$ & $84^{\mathrm{e}}$ & $96^{\mathrm{e}}$ & $100^{\mathrm{e}}$ \\
\hline
\end{tabular}

Tableau 8

Les facteurs d'indécision dans l'enseignement secondaire ( $\mathrm{N}=1152)$

\begin{tabular}{|l|c|c|c|c|c|c|c|c|c|c|}
\hline Classes & 1 & 2 & 3 & 4 & 5 & 6 & 7 & 8 & 9 & 10 \\
\hline Information & $8-11$ & $12-14$ & $15-16$ & $17-18$ & 19 & $20-21$ & $22-23$ & $24-25$ & $26-27$ & $28-32$ \\
\hline Soi & $8-9$ & $10-11$ & $12-13$ & $14-15$ & $16-17$ & 18 & $19-20$ & $21-22$ & $23-26$ & $27-32$ \\
\hline Méthode & $8-10$ & $11-12$ & $13-14$ & $15-16$ & $17-18$ & $19-20$ & $21-22$ & $23-24$ & $25-27$ & $28-32$ \\
\hline Développement & 8 & 9 & 10 & $11-12$ & 13 & $14-15$ & $16-17$ & $18-21$ & $22-32$ \\
\hline Anticipations & $8-11$ & $12-13$ & $14-15$ & $16-17$ & 18 & $19-20$ & $21-22$ & $23-24$ & $25-26$ & $27-32$ \\
\hline Obstacles & \multicolumn{1}{|c|}{8} & & 9 & 10 & 11 & 12 & 13 & $14-32$ \\
\hline
\end{tabular}




\section{BIBLIOGRAPHIE}

Forner, Y. (1995). L'indécision vocationnelle à la fin du collège. Document de travail. Lille: Université Lille 3 et Paris: INETOP. Document ronéoté.

Forner, Y. (1999). Épreuve de Décision Vocationnelle. Paris: E.A.P.

Forner, Y. (2001). À propos de l'indécision. Carriérologie, 8(2), 183-194. (www.carriero logie.uqam.ca/)

Forner, Y. (2007). L'indécision de carrière des adolescents. Le Travail Humain, vol. 70, $n^{\circ}$ 3, 213-234.

Forner, Y. (2009). L'Échelle de Décision Vocationnelle. Formes S et I.Manuel. Paris: Éditions Eurotests.

\section{ANNEXES}

Annexe 1 : Distributions des scores aux items

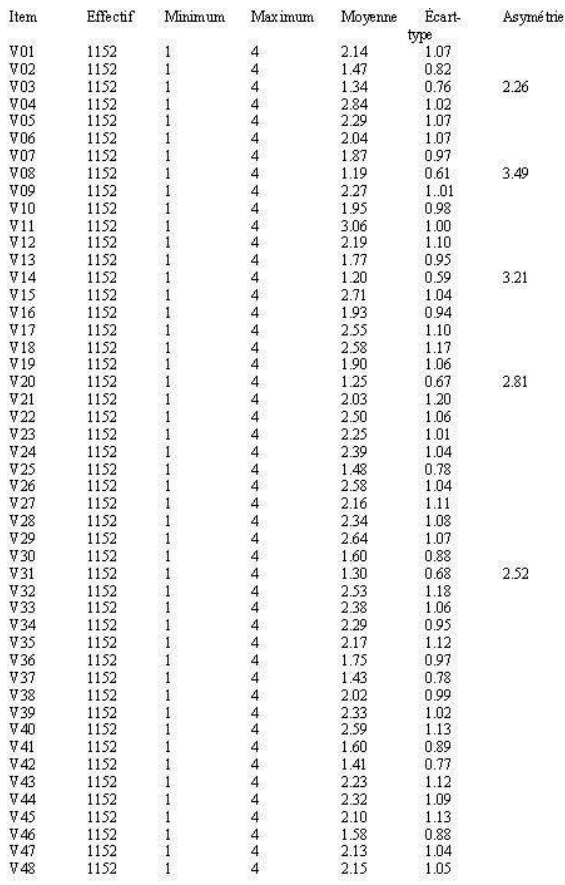

Annexe 2 : EDV-95 - Échelle de Décision Vocationnelle (forme S) 


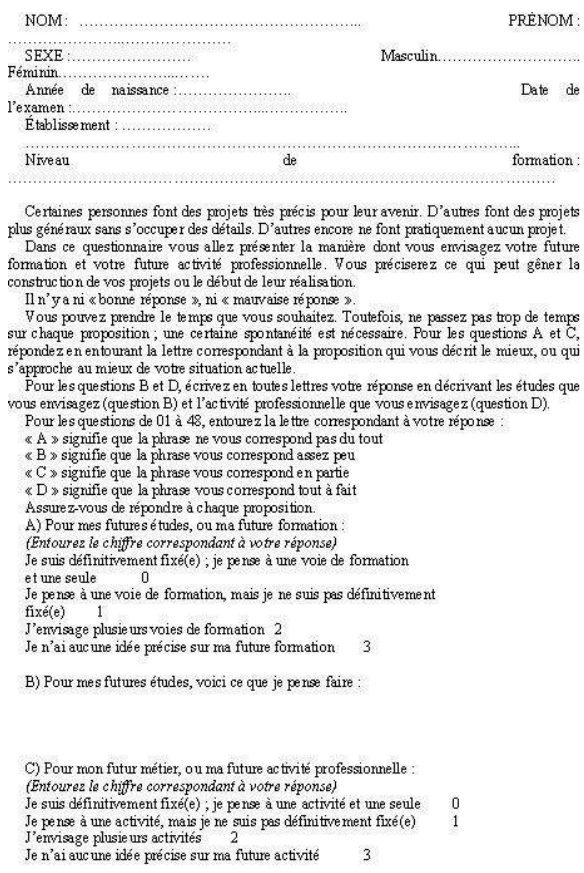

\section{RÉSUMÉS}

L'indécision vocationnelle désigne l'incapacité à faire un choix en matière de formation ou d'emploi quand celui-ci s'avère nécessaire. Les causes potentielles de cet état indécis sont nombreuses et il est important de les recenser pour préciser la forme à donner au conseil en orientation. C'est ce que permet l'EDV-9, une nouvelle version du questionnaire EDV. La nouvelle épreuve est présentée de manière détaillée : fondements théoriques, évolution du questionnaire, qualités psychométriques et étalonnage. Le questionnaire EDV-9 (forme $S$ ) est fourni.

Vocational indecision refers to the inability to make a choice about a major or a vocation, when a choice has to be made. Possible determinants of this undecided state are numerous and must be reviewed before specifying what sort of guidance is required. That is what EDV-9, a new form of the EDV inventory, is designed to. This new test is detailed: theoretical foundations, variations of the inventory, psychometric properties, and sampling - EDV-9 (S form) questionnaire is provided.

\section{INDEX}

Keywords : Assessment, Career, Decision-making, Indecision, Vocational guidance

Mots-clés : Carrière, Ėvaluation, Indécision, Orientation professionnelle, Prise de décision

\section{AUTEUR}

\section{YANN FORNER}

est Professeur de psychologie à l'Université de Lille 3 et responsable du Centre de formation de conseillers d'orientation - psychologues de Lille. 